\section{Palobra}

acceso $\odot$ abierto

Cómo citar: Pineda, Y. y Loaiza, Y. (2020). Prácticas pedagógicas del maestro de las escuelas normales del Eje Cafetero, 19942010. Palobra, 20(2), 192-211.

10.32997/2346-2884-vol.20-num.2-2020-3315

Recibido: 5 de junio de 2020 .

Aprobado: 21 de septiembre de 2020 .

Autor de correspondencia:

Yheny Lorena Pineda-Rodríguez

yhelopi@gmail.com

Editora: Rosario Blanco Bello. Universidad de Cartagena-Colombia.

Tipología IBN Publindex:

Artículo de Investigación Científica.

\title{
Prácticas pedagógicas del maestro de las escuelas normales del Eje Cafetero, 1994-2010
}

\author{
Pedagogical practices of teachers in the teacher training schools \\ of the Eje Cafetero region, 1994-2010
}

\author{
Yheny Lorena Pineda-Rodríguez \\ Universidad de Caldas, Colombia, yhelopi@gmail.com \\ Yasaldez Eder Loaiza-Zuluaga \\ Universidad de Caldas, Colombia, yasaldez@ucaldas.edu.co
}

\section{RESUMEN}

El presente artículo tiene como objetivo presentar los resultados de la investigación denominada Prácticas pedagógicas que caracterizaron el quehacer del maestro en el Ciclo de Formación Complementaria (CFC) de las Escuelas Normales del eje cafetero, 1994-2010. Investigación que se adelantó en el campo de la historia de la educación, desde lo cualitativo, con un enfoque histórico hermenéutico, que utiliza como técnica de recolección de información la entrevista narrativa con los maestros formadores de maestros y la revisión documental de archivos históricos. Luego de analizar los datos se ha encontrado que, desde la categoría bourdieana habitus, se evidencian extrínsecamente prácticas pedagógicas determinadas por la máxima autoridad pedagógica, legitimadas desde los ámbitos político y legislativo; e intrínsecamente desde la normatividad institucional de las escuelas normales y desde el sistema integrado de quehaceres configurado en torno al discurso frente al saber pedagógico y a las realidades del mismo en los diversos escenarios pedagógicos. Se concluye que, aunque las Escuelas Normales en las últimas tres décadas han tenido procesos diferenciados de transformación, las reestructuraciones para certificarse en los diferentes procesos de acreditación, implicaron prácticas pedagógicas particulares -sin determinar su efectividad o desacierto- que correspondieron a un momento específico del desarrollo de la escuela y de la relación de la escuela con la sociedad.

Palabras clave: Escuelas normales; práctica pedagógica; quehacer; maestro; habitus.

\footnotetext{
ABSTRACT

The aim of this article is to present the results of the research called Pedagogical Practices that characterized the work of the teacher in the Complementary Training Cycle of the Teacher Training Schools of the Coffee Axis, 1994-2010. Research that was advanced in the field of the history of education, from the qualitative, with a

${ }^{1}$ Este articulo presenta los resultados de la investigación denominada Prácticas pedagógicas que caracterizaron el quehacer del maestro en el Ciclo de Formación Complementaria (CFC) de las Escuelas Normales del eje cafetero, 1994-2010. Investigación financiada por el Departamento Administrativo de Ciencia, Tecnología e Innovación Colciencias - Colombia, código 785 de 2017, Doctorados Nacionales. Vicerrectoría de Investigaciones y Posgrados, Universidad de Caldas.
} 
hermeneutic historical approach, which uses as a technique of information gathering the narrative interview with teacher trainers and the documentary review of historical archives. After analyzing the data, it has been found that, from the Bourdieana habitus category, pedagogical practices determined by the highest pedagogical authority, legitimized from the political and legislative spheres, are extrinsically evident; and intrinsically from the institutional normativity of the normal schools and from the integrated system of tasks configured around the discourse before the pedagogical knowledge and the realities of the same in the different pedagogical scenarios. It is concluded that, although the Teacher Training Colleges in the last three decades have had different transformation processes, the restructuring to be certified in the different accreditation processes, involved particular pedagogical practices -without determining their effectiveness or inadequacy- which corresponded to a specific moment in the development of the school and the relationship of the school with society.

Keywords: Teacher training schools; pedagogical practice; teacher; habitus.

\section{INTRODUCCIÓN}

El objetivo del presente artículo de investigación, es presentar los resultados de la investigación doctoral denominada Prácticas pedagógicas que caracterizaron el quehacer del maestro en el Ciclo de Formación Complementaria de las Escuelas Normales del eje cafetero, 1994-2010, a partir del cual se evidenció la configuración de la práctica pedagógica, desde un sistema integrado en torno a la tríada discurso pedagógico, acción pedagógica desde el quehacer del maestro en un escenario pedagógico o escuela normal.

El texto, aborda históricamente conceptos vinculados con la práctica pedagógica con relación a la misión pedagógica de las normales y que se van transformando a medida que cambian los enfoques pedagógicos, la acción pedagógica institucional, el trabajo pedagógico de inculcación rutinizada del maestro, el habitus, el sentido práctico y la investigación; análisis propuestos desde sustentos teóricos de la teoría de Bourdieu con relación al concepto de habitus como categoría central, a los que se vinculan, narraciones de los sujetos del saber pedagógico de las normales del eje cafetero.

De manera que, el saber pedagógico, didáctico y curricular, fue evidente desde lo declarativo, desde la normatividad y reglamentación institucional, el PEI; las realidades del saber se acreditaron en lo procedimental del trabajo pedagógico de los maestros y en la acción pedagógica institucional de cada escuela normal; evidentes, a su vez, en los diversos escenarios pedagógicos, los que conjuntamente se regularon a partir de la legislación vigente en el período de estudio; advenimientos que la caracterizaron y que, a su vez, fueron transmitidos a los educandos en formación. 
Para comprender cuáles fueron las prácticas pedagógicas que caracterizaron el quehacer del maestro en el CFC de las Escuelas Normales del eje cafetero, es necesario abordar dos categorías que, en conjunto evidencian las prácticas pedagógicas del sujeto del saber pedagógico: primero, desde el deber ser del maestro derivado de las exigencias propias del campo cultural, del campo educativo colombiano con miras a la reestructuración de estas instituciones formadoras de maestros, producto del período del movimiento pedagógico (Pineda-Rodríguez y Loaiza-Zuluaga, 2017), que otrora favoreció la lucha por la reivindicación laboral y pedagógica de los maestros, para conseguir un estatus en el campo cultural, en el campo educativo.

Y segundo, eje central del presente análisis y que se desarrollará con mayor fuerza, desde el concepto de habitus, como estructuras que se incorporaron institucionalmente, desde la acción pedagógica colectiva y, particularmente, desde el trabajo pedagógico de inculcación rutinizada, en el quehacer del maestro y que, finalmente, caracterizaron la realidad del objeto de estudio, las prácticas pedagógicas.

Prácticas pedagógicas que transitaron inicialmente, por un enfoque tradicionalista, reproductor, a principios de la década del noventa del siglo XX (Loaiza, Pineda y Arbeláez, 2014), hacia otras prácticas pedagógicas, algunas con características y principios que permanecieron en el fondo de las estructuras de los habitus productores de práctica pedagógicas, cultivadas e incorporadas, con el fin de mantenerse vigentes; o bien, prácticas pedagógicas mediadas por el sentido práctico del maestro o la toma de decisiones con base en su experiencia; o prácticas pedagógicas distintas, que, hacia finales de la primera década del siglo XXI, se caracterizaron por partir de enfoque por procesos, problematizador, a partir de núcleos temáticos diferenciados, de transformación, que implicaron reflexiones de la teoría, la práctica y la investigación, al integrar elementos propios de la investigación educativa.

Entre tanto, el ejercicio de aplicación de la pedagogía, como reflexión intencionada de la interacción simbólica y cultural, y de su respectiva reestructuración dada por el saber pedagógico, estuvo reflejada en la denominada práctica pedagógica, práctica determinada según la formación del maestro, pero también con base en condiciones, circunstancias y necesidades de la realidad educativa existente, que caracterizaron el sujeto del saber pedagógico, como la mediación articuladora entre el campo del conocimiento y el campo del trabajo, entre la teoría y la práctica (Ávila, 2007), entre lo extrínsecamente dispuesto y lo intrínsecamente favorecido.

\section{Metodología}

Iluminó la investigación, la concepción de investigación histórico-educativa con un hilo historiográfico, que esbozó el diseño de la investigación desde el Trayecto como fundamento del método histórico (Loaiza, Pineda y Arbeláez, 
2014; Valencia, 2006), que permitió comprender los contextos sociales, políticos y educativos que particularizaron la época.

En consecuencia, las cuatro etapas que configuraron el diseño de la investigación fueron, primero la fase Heurística o recopilación de los datos documentales (Cardoso, 2000); segundo, la fase analítica u organización, sistematización y tratamiento de esos datos a partir del control cruzado entre diferentes fuentes, denominada doxografía; tercero, la interpretación histórica de todo el material primario mediante una exposición ordenada y sistemática para convertirlo en historia a través del relato, también llamado etiología "descodificación" o "transcodificación" (Cardoso, 2000); y finalmente, la síntesis histórica como la síntesis dialéctica, que trata de vincular en una visión unificada, los enfoques estructural y genético.

La unidad de trabajo estuvo determinada por cuatro escuelas normales superiores de ciudades capitales del eje cafetero. Las técnicas e instrumentos cualitativos que contribuyeron a la recolección de información, a partir de las cuales se estructura el presente informe, fueron, primero, el análisis de documentos primarios, que permitió la triangulación de fuentes a partir de la información recolectada, procedente de historiales, manuales, informes de rectores, supervisores y pares académicos, actas de reuniones y de visitas, correspondencia, oficios despachados y recibidos, cartas, circulares, artículos de prensa, textos, libros, escritos y memorias, entre otros, localizadas principalmente, en los archivos de las escuelas normales del eje cafetero, relacionados con las prácticas pedagógicas que caracterizaron el quehacer de los maestros de estas instituciones.

Segundo, la sistematización de 16 entrevistas en profundidad y cuatro grupos focales, aplicados a los actores del saber pedagógico, desde el análisis del discurso, de las cuales se toman algunos apartados, con el propósito de ilustrar los hallazgos en el presente artículo; para determinar la unidad de análisis, se tuvo como criterio de inclusión, maestros que hubiesen laborado en el denominado CFC de la escuela normal superior, en el período de la investigación 1994-2010.

\section{Construcción de sentido: Hallazgos}

Las prácticas pedagógicas que caracterizaron el quehacer de los maestros en el CFC, entre 1994 y 2010, fueron comprendidas, a partir de la identificación de los postulados que emergieron de la normatividad de la época y de la interpretación en torno a los discursos, las acciones y los escenarios pedagógicos que identificaron el maestro formador de maestros. La sistematización de los datos recolectados, su análisis con base en la codificación y categorización, así como la interpretación de los mismos, fue posible, desde la relevancia de los datos hasta determinar la estructura de 
comprensión con relación a la categoría prácticas pedagógicas y al concepto de habitus.

La autoridad pedagógica o Ministerio de Educación Nacional (MEN), en Colombia, a través de las políticas educativas que legitimaron el sistema educativo, ha monopolizado la producción de agentes, de maestros, encargados de reproducir, con todo lo que está asociado a ello, lo que se constituyó, en el momento histórico de la presente investigación, como cultura legítima. Ahora bien, cada contexto educativo, en este caso, cada escuela normal superior del eje cafetero, propició, desde las "características específicas de su estructura y de su funcionamiento", una acción pedagógica institucional, a través del quehacer del maestro o del llamado trabajo pedagógico, colectivo e individual, de los sujetos del saber pedagógico de inculcación por parte de cada maestro formador de maestros.

En ese sentido, se evidenció cómo la acción pedagógica institucional dependió, extrínsecamente, con relación a las prácticas pedagógicas, de la máxima autoridad pedagógica o MEN, legitimada desde los ámbitos político y legislativo que marcaron el rumbo de estas instituciones formadoras de maestros, al determinar para ellas, a lo largo del período de estudio, procesos diferenciados de transformación y reestructuración para certificarse en los diferentes procesos de acreditación previa y acreditación de calidad, que implicaron prácticas pedagógicas particulares -sin determinar su efectividad o desacierto- según los desarrollos de las mismas y de la relación de la escuela con la sociedad.

De ahí que, la formación de maestros atendió y consideró las políticas nacionales e internacionales relacionadas con el mejoramiento de la calidad de la educación, con los procesos implicados en el aprendizaje y con el componente emocional; aprendizaje visto desde una perspectiva de construcción personal de conocimiento y enseñanza como una acción generada por los maestros, en las normales, a través del trabajo pedagógico de inculcación rutinizada, para conseguir las intenciones educativas en cada uno de sus estudiantes. Fue en el campo de la práctica donde se afirmó, que los maestros en formación descubrieron la pedagogía (Sáenz, 2010).

En consecuencia, la autoridad pedagógica o MEN, tuvo injerencia en la instancia pedagógica o secretaría de educación, y esta a su vez, en la acción pedagógica de cada normal, legitimado desde la normatividad institucional, viabilizado en el trabajo pedagógico, en el quehacer de sus agentes o maestros $\mathrm{y}$, finalmente, en sus prácticas pedagógicas, bajo los principios de la arbitrariedad que ordenaron la práctica, la naturalizaron y convirtieron en un esquema productor de prácticas, en un mensaje escolar codificado, homogéneo, sistematizado y ortodoxo, integrado en una cultura rutinizada (Bourdieau y Passeron, 1996). Al respecto, el siguiente apartado lo ejemplifica: 
En Armenia el plantel docente depende de la secretaría de educación, entonces la secretaría depende del secretario que toma la decisión de decir si apoya o no; acá por fortuna siempre se apoya. La normal tiene los dos secretarios, el secretario municipal y secretario departamental; el rector es de la secretaría municipal y el coordinador del secretario departamental (A, Angulo, comunicación personal, julio de 2018).

El trabajo pedagógico, colectivo e individual de los maestros, implicó, además de tener un público legítimo de estudiantes y padres de familia, la inculcación rutinizada del trabajo sobre el pedagogo, sobre el maestro formado para ello, capaz, pero, posiblemente, desconocedor de los principios de su realización, de la condición de violencia simbólica original, arbitraria y de la delegación de la autoridad pedagógica de ese poder arbitrario al ser producto del sistema que lo formó, por tanto tenía una conciencia acotada de la relativa inconsciencia del porqué hacía lo que hacía (Bourdieau y Passeron, 1996). Un ejemplo de ello:

Cuando yo llegué a la normal ya estaban ahí propuestos los temas y los contenidos; la profe me dijo, vea, acá están los contenidos, luego fue evolucionando. En el programa cuando yo llegué recibí unas asignaturas: Administración Educativa, entonces porque me dieron administración fue porque yo tengo un estudio en técnica en administración y en mercadeo; yo tenía ese tipo de experiencia, entonces empecé a trabajar, entonces habían eran compendios, temas, generalidad de administración (A. Angulo, comunicación personal, julio de 2018).

Siguiendo a Bourdieau y Passeron (1996): "los agentes, dotados de la formación duradera, les permitió mantener y reproducir, en el tiempo, las condiciones institucionales del ejercicio" (p. 110) con base en reglas formales y normas explícitas propias de cada institución, de cada contexto, que tendieron a reproducir, desde su quehacer, desde la inculcación rutinizada del trabajo pedagógico, esa misma "formación en nuevos reproductores y por ello encerró una tendencia a la autorreproducción perfecta o inercia, que se ejerció en los límites de su autonomía relativa" (Bourdieau y Passeron, 1996, p. 110).

\section{Discusión}

\section{Práctica pedagógica de maestros formadores de maestros: 1994-2010.}

A principios de la década del noventa se reconocieron dos enfoques que caracterizaron el quehacer del maestro del CFC y, por ende, las prácticas pedagógicas en torno a la formación del mismo. El primero, tradicional, legitimado desde la normatividad institucional, con procesos de aula donde el maestro a través de sus prácticas pedagógicas fue el centro del proceso educativo, "el maestro hablaba y el alumno escuchaba" (Pineda-Rodríguez y Loaiza-Zuluaga, 2017, p. 164), encargado desde su discurso, de explicar, 
demostrar, preguntar, colocar tareas, calificar, promover con base en las notas obtenidas por el alumno sobre los temas del programa oficial; y el alumno, centrado en atender, estudiar, hacer tareas, responder lecciones, previas y exámenes, aprobar o perder con base en las notas obtenidas sobre los temas enseñados por el profesor (Cajamarca, 1992). El siguiente testimonio, lo ejemplifica:

Recuerdo mucho la disciplina de mi Generala Chacón. Hoy digo gracias a Dios tuve esos maestros que se preocuparon por la disciplina, porque yo llevará una falda donde la debía llevar porque había mucho conductismo y yo trato de no ser tan conductual (L. Aguilar, comunicación personal, julio de 2018).

El segundo, el enfoque por procesos, donde el maestro deja de ser el centro de la clase y pasa a ser quien, a través de sus discurso y acción, orienta, asesora, dirige y coevalúa el cómo y el qué debía aprender el alumno con base en el proceso mental, afectivo, volitivo y psicomotor de los contenidos curriculares del aprendizaje; y el estudiante, pasa a ser el protagonista, quien aprende contenidos curriculares mediante el desarrollo personal de los procesos de formación mental, afectiva, volitiva y psicomotriz, promovido con base en la autoevaluación de los contenidos de aprendizaje. Este último, caracterizado por la jerarquización de responsabilidades en la formación integral de los procesos, así el primero era el educando, el segundo los padres de familia y el tercero el centro educativo (Cajamarca, 1992).

Posteriormente, cada maestro formador del CFC, inscrito en un enfoque y núcleo temático, según su especialidad, contó con tres características fundamentales en su quehacer, para alcanzar los objetivos propuestos y legitimados desde la normatividad institucional, entre los que estuvieron la comunicación colectiva permanente entre los maestros que componían el núcleo para unificar criterios, la evaluación del mismo y el estudio permanente. Fue así como se consideró que los contenidos fueran los medios para alcanzar los logros y que el profesor, de manera individual, tenía el dominio general, el saber pedagógico de todos los aspectos que componían el núcleo temático, en concordancia con las disposiciones del grupo, institucional o en convenio (Escuela Normal Superior de Manizales, 1997). El siguiente testimonio, lo ilustra:

Nosotros trabajábamos con otras guías que eran de Carlos Cajamarca... era para la básica, la media y el CFC. En ciclo, teníamos unos módulos con las guías, nosotros tuvimos que hacer sin saber cómo se hacía un módulo, todo empezar a machar. La Secretaría de Educación del Departamento asumió ese compromiso, hicieron un convenio con las universidades que tenían facultad de educación, pero ellos también estaban aprendiendo el cuento de los módulos y eso, ellos tampoco sabían, ahí fue cuando nos unimos con ellos en grupos de trabajo y empezamos a crear cosas de esas (A. Salazar, comunicación personal, julio de 2018). 
En ese sentido, cada maestro del CFC aplicó a sus estudiantes categorías de percepción, acción y pensamiento con base en los habitus o las estructuras estructuradas en las que él fue formado, así como con las nuevas incorporaciones o actualizaciones de las prácticas producto del habitus incorporado. Por tanto, la acción pedagógica institucional de la escuela normal fue el resultado de las prácticas pedagógicas que cada maestro incorporó a sus estudiantes mediante habitus dispuestos, los que a su vez fueron el resultado de los habitus incorporados en los que el mismo maestro fue formado conjuntamente con su evolución, involución, transformación y/o actualización (Bourdieu, 1997).

El maestro del CFC, incluyó la inculcación rutinizada del trabajo pedagógico, del sistema de enseñanza desde contenidos pedagógicos, didácticos y hasta investigativos, bajo el axioma "lo que se deja de hacer a través del trabajo pedagógico o la Normal [desde la acción pedagógica], luego no podrá ser aplicado por los estudiantes y/o por los maestros" (Nieto, 1998, p. 2). Así pues, no fue extraño encontrar algunos maestros jóvenes, resultado de habitus incorporados, reacios al cambio, a la investigación y a la participación, en razón a que carecían de aprendizajes, discursos y experiencias vividas en procesos institucionales; por lo tanto, los nuevos maestros, fueron temerosos en su quehacer para aplicar lo que desconocían, dado que fueron, posiblemente, reprimidos en el proceso de su formación.

Al respecto, con base en los postulados de Bourdieau y Passeron (1996), se podría afirmar que las prácticas de los maestros obedecieron, a la "cultura de la rutinización, al habitus incorporado que legitimó la arbitrariedad impuesta” (p. 101), al estar definida esta categoría en el trabajo pedagógico, por su posición en el sistema de enseñanza institucionalizado desde la normatividad propia de cada normal; y, contrariamente, cuanto más participaron en "otros campos de práctica como por ejemplo en el campo científico o intelectual" (Bourdieau y Passeron, 1996, p. 101), en la reflexión teoría y acción, más alejado podría estar su quehacer de la arbitrariedad legítima impuesta. El siguiente testimonio, así lo manifiesta:

Y llegó pues y todo, ya empezaron las críticas y empezaron, ah ya, hay muchas actitudes en contra de nosotros, en contra de nosotros y eso se volvió como personal, pasó de ser profesional, ya, mejor dicho, personal era que ya no nos hablábamos, nos hacían mala cara, mejor dicho, sátiras, nos decían sobrenombre los mismos compañeros de la institución: claro, ya nos van a quitar el puesto, se creen que saben lo que no se... pero nosotros empezamos a hacer análisis de las teorías, reflexiones, mejor dicho, ahí entendimos la vinculación entre teoría y práctica, ya entendimos que el trabajo del maestro era reflexión, acción, indagación y propuesta. Entonces nosotros empezamos a cambiar muchas cosas, cambiamos las metodologías, flexibilizamos ya, por ejemplo, en el aula de clase, por ejemplo, la disciplina, en el aula de clase ya no era de regañar o desacreditar [...] Y ahí cuando nosotros cambiamos la mayoría de los métodos tradicionales que estábamos 
ubicados en la técnica o la formulita. Nosotros teníamos, por ejemplo, en la anexa, a los maestros de formación, lo que les dábamos era formulitas, usted va a....Teníamos pasos [habituales] para todo, el paso para la matemática son estos dos pasos, el paso para la lectura y escritura son estos pasos, el paso para las ciencias naturales son estos pasos, tenemos fórmulas para cada una de las áreas, y cuando nosotros empezamos, esas fórmulas desaparecieron. (L. Cortés, comunicación personal, julio de 2018).

Fue así como, a finales de la década del noventa, el maestro continuó desempeñándose como orientador del núcleo básico de trabajo correspondiente a su especialidad, acorde con el número de logros del nivel ubicado. Legítimamente, su práctica pedagógica y su discurso, se evidenciaron en la planeación, organización y control de los núcleos básicos del trabajo pedagógico, de acuerdo con el desarrollo curricular, en el cual procuró identificar las necesidades de formación de los futuros maestros; pretendió instaurar mecanismos de seguimiento para descubrir fortalezas y posibilidades en los maestros en formación; se comprometió con la utilización de estrategias de apoyo pedagógico; colaboró en el proceso de inserción del maestro en formación en la estructura educativa propia de la época; introdujo y aplicó diferentes criterios de evaluación; fijó canales de información que ayudaron a obtener la comunicación que se necesitaba; le correspondió determinar los recursos de apoyo que consideró necesarios en cada actividad docente; y formó parte del colectivo docente que evaluó y apoyó la práctica (Jaramillo, Candamil y Ocampo, 1999).

Justamente, fue el habitus "por medio del aprendizaje implícito o explícito, de las posibilidades e imposibilidades, de las libertades y necesidades, de las facilidades y prohibiciones" (Bourdieu, 1993, p. 94) inculcado por los maestros formadores de maestros, el que funcionó como un "sistema de esquemas generadores de nuevos habitus, inscritos en las constricciones y límites objetivos, incorporados y regulados, conformes con los intereses objetivos de sus autores" (Bourdieu, 2002, p. 125), los que, para el caso de las normales, fueron los maestros y las directivas.

En consecuencia, el maestro a través del habitus o sistema de disposiciones socialmente dispuestas e incorporadas desde estructuras previamente estructuradas, estructuró prácticas pedagógicas legítimas, productoras de otras prácticas pedagógicas e ideologías frente a la formación de maestros, característicos del grupo de agentes, maestros formadores. Tales disposiciones se tradujeron en actos incuestionables, axiomáticos, individuales y/o colectivos, legitimados en la normatividad institucional y viabilizado al interior del campo intelectual, que a su vez ocupó una posición precisa en la estructura del capital simbólico dominante (Bourdieu, 2002). El siguiente fragmento, así lo ejemplifica: "Tengo la emoción de que incluso tengo ahí sentado uno de los que yo formé en la primera promoción que yo estuve como maestra formadora, ahí ya está el chico sentado a la par con 
uno, igualito a uno” (G, Galindo, comunicación personal, julio de 2018).

Para ilustrar el tránsito entre habitus rutinizados y habitus estructurantes de otras prácticas pedagógicas, las reflexiones particulares de la Escuela Normal Superior El Jardín de Risaralda son un buen ejemplo, dado que, como bien lo reflejaron sus discursos con relación al saber pedagógico, mantuvo hasta principios del siglo XXI, una acción con pocos cambios frente al enfoque pedagógico tradicional, a pesar de las variaciones en los conceptos de enseñanza, aprendizaje y formación, que promovían no enseñar lo mismo y de la misma forma todo el tiempo (Ocampo y Espitia, 2001).

Con relación al concepto de formación, desde el discurso, dependió, en principio, de los habitus incorporados en la escuela que formó al maestro del CFC, así como de la tendencia pedagógica que diferenciase su quehacer o trabajo pedagógico (Ocampo y Espitia, 2001). En ese sentido, es posible afirmar que las disposiciones incorporadas o habitus, crearon en los maestros el denominado por Bourdieau (1997), el sentido práctico, es decir la toma de decisiones anticipadas con base en sentimientos, preferencias, decisiones, principios, visiones y comprensiones del mundo, como resultado de las disposiciones y estructuras incorporadas en la institución donde fue formado, es decir, desde la escuela normal, que llevó al maestro a desempeñarse de una u otra manera con base en actuaciones objetivas y legítimas dentro del campo cultural o campo educativo al que pertenecían sus prácticas pedagógicas (Bourdieu, 1997). El siguiente fragmento de un sujeto del saber pedagógico, así lo indica:

Cuando uno es docente, primero es un orgullo y un privilegio, eso implica que somos toderos, o sea, no hacemos una cosa sino una, dos, tres, cuatro, cinco, seis. Nosotros los maestros cantamos y silbamos y hacemos todo lo que nos pongan, cierto, somos toderos; además que somos el referente institucional cuando hay capacitación, los primeros que estamos ahí frente al cañón; somos los del programa de formación y siempre hacemos capacitación entonces, mira que es una responsabilidad bien fuerte (C. Marín, comunicación personal, julio de 2018).

A principios del siglo XXI, se afirmó, desde la normatividad, que la práctica pedagógica en las escuelas normales estaba estructurada por núcleos temáticos, instituidos en el Proyecto Educativo Institucional (PEI), desde la teoría pedagógica hasta la teoría práctica, a la que se le adicionó un nuevo elemento, la investigación, para confrontar la teoría con la práctica. Fue necesario, una mentalidad abierta, sin concepciones a la fuerza, de cambio a través del habitus incorporado, tanto para los maestros como para los directivos y administrativos para lograr, paulatinamente, este propósito, desde lo macro como acción pedagógica institucional y, desde lo micro, a partir de la inculcación rutinizada del trabajo pedagógico, el quehacer individual y colectivo. En ese sentido, las normales fueron consideradas los espacios de 
estudio de los conocimientos disciplinares y pedagógicos que debieron estar en proceso de construcción y reconstrucción permanente, interactuando con el conocimiento y con el saber (Ocampo y Espitia, 2001). El siguiente testimonio, lo ejemplifica:

Cuando yo llegué la Normal, tenían un ambiente académico, la Normal estaba dividida en dos; unos, uno era toda esa generación ... un grupo de personas intelectuales, académicos con proyecto institucional que lo lideraron, entonces estos querían la transformación de la escuela ... y estaba el otro grupo, empoderado en su zona de confort. Una incisión ahí ... establecer alianzas para ganar una guerra y este grupo desapareció con el tiempo [...] hay que hacer esto, hay que hacer esto ... no resistían, era una lucha subterránea, eso fue muy fuerte .... Y sabiendo que había una empatía académica (L. Uribe, comunicación personal, julio de 2018).

Conjuntamente, la práctica pedagógica se asumió como el conjunto de acciones entre los sujetos, sus discursos y la cultura, mediados por el PEI, en un contexto particular. La práctica y la investigación pedagógica se establecieron como factores imprescindibles en la formación de los maestros, al difundir un espíritu investigativo en maestros y estudiantes y crear en ellos, espacios de reflexión sobre su formación y su quehacer; sin embargo, se observó, según el MEN (citado por Lloreda, 2002, p. 5), una desarticulación entre la práctica pedagógica de los futuros maestros y el desarrollo de procesos de investigación, tal como lo exigía la normatividad nacional. En efecto, al constatar el quehacer del estudiante de $\mathrm{CFC}$ en la práctica pedagógica, se evidenciaron inconsistencias entre el discurso pedagógico y la acción pedagógica, puesto que evidenciaron características del enfoque tradicional, con reproducción del conocimiento, tanto en maestros formadores como en maestros en formación (MEN, 2002).

La práctica pedagógica, estuvo definida teórica y conceptualmente desde el modelo pedagógico de cada escuela normal, en su PEI, viabilizada por el trabajo pedagógico del maestro, desde la inculcación rutinizada del habitus y por la acción pedagógica de la institución. En consecuencia, dada su misión pedagógica, fueron consideradas las normales, laboratorios pedagógicos, escenarios donde los futuros maestros encontraron el referente a su futura actuación, en la formación de ciudadanos; con teorías y discursos pedagógicos claves para un aprendizaje exitoso a través del quehacer del maestro. Se afirmó que "el éxito de la formación, no la instrucción sino la exigencia permanente, surge del ejemplo" (Escuela Normal Superior de Manizales, 2002, p. 2).

Misión pedagógica que fue leída e interpretada por agentes externos a las normales, legitimados por el MEN, con el fin de recolectar evidencias que dieran cuenta de los procesos de la Normal en la formación de maestros. Fue así como, con base en la legislación nacional para la formación de maestros, 
aplicaron diversos instrumentos de recolección de información para contrastar dichos procesos, en las normales del eje cafetero y del país; entre ellos contaron con la observación directa de los diferentes escenarios educativos, del quehacer del maestro y sus prácticas pedagógicas en las diferentes aulas de los niveles ofrecidos en la institución; así como diálogos abiertos con los actores, reuniones con grupos académicos del gobierno escolar y comunidad; visitas a laboratorios, bibliotecas, salas de computo, centros educativos de prácticas pedagógicas e investigativas y otros ambientes de la vida institucional; además del análisis documental de los archivos académicos, de tesorería y de bienestar, reflejo del discurso y saber pedagógico de los colectivos docentes (López, Tobos y Baldovino, 2003).

Así, según el informe de la Evaluación Externa de Acreditación, se denotó en la Escuela Normal Superior de Risaralda un proceso de construcción, de comunidad académica, donde el maestro se apropió de un saber, asumido como un recurso que se sustentó en los discursos pedagógicos y se reflejó en el quehacer, a través de las prácticas pedagógicas fundamentadas en principios y postulados propios de la propuesta de formación, con base en la normatividad institucional. En conclusión, se observó claridad tanto en su discurso como en las prácticas pedagógicas del aula (López, Tobos y Baldovino, 2003). El siguiente testimonio, así lo refleja: "Cuando yo llegué por ejemplo a la Normal, yo llegué y la Normal tenía su construcción del PEI, lo que se hacía era simplemente mejoras, lo que se hacía era simplemente situaciones” (G. Mendieta, comunicación personal, septiembre de 2018).

El siguiente testimonio, lo ejemplifica:

ASONEN jugó un papel importante durante el proceso como de acreditación de alta calidad y en esa época hubo unos congresos rebuenos, yo me acuerdo que en Medellín hubo uno súper chévere vinieron normales de México, Venezuela, porque en Venezuela se habían acabado las normales y estaban en proceso de volverlas a crear; vinieron de Chile, de Argentina y vino el Instituto Pedagógico de París y trajeron un señor un viejito, un profesor que era pues mejor dicho el que más sabía, el gurú de la pedagogía, de quien era que se llamaba... ese pedagogo francés Declaparede yo creo que era ese, vino y revolucionó el cuento (A. Salazar, comunicación personal, julio de 2018).

Entre tanto, para garantizar la misión de formación de maestros legitimada desde la normatividad nacional, la Escuela Normal Superior de Manizales, normalizó la acción pedagógica institucional desde la comunidad académica, coordinada por el comité de práctica pedagógica, encargado de la toma de decisiones que incidieron, directamente, en la inculcación rutinizada del trabajo pedagógico de los maestros, específicamente en lo que denominaron práctica pedagógica investigativa, tanto para los maestros formadores de maestros como para quienes se formaron para serlo. Fue así como se 
determinó para los maestros en formación, la asignación de dos maestros asesores del proyecto de investigación, uno de práctica y un técnico de la básica secundaria y media, quienes, a través de sus prácticas pedagógicas, mejorarían la calidad de quehacer pedagógico e involucrarían a todo el cuerpo docente en actividades conjuntas al CFC (Escuela Normal Superior de Manizales, 2004).

Y fue precisamente, el campo cultural, el que propuso a los agentes o maestros que conformaron la planta docente con un universo de problematizaciones legítimas, de referentes teóricos que fundamentaron las bases de sus habitus y por ende de sus prácticas, con conceptos que inculcaron y cimentaron la rutinización de los principios de sus estructuras incorporadas y que dieron como resultado determinadas prácticas pedagógicas (Bourdieu, 1997). El siguiente testimonio, lo ejemplifica:

\begin{abstract}
Recuerdo que manteníamos en pleno estudio con una profesora llamada Patricia, mirando la malla curricular y con el rector que, en su sabiduría, era el que más nos daba pedagogía, entonces siempre que nos llamaba era, a decirnos desde donde enfocamos y estábamos en el estudio, también de lo que era nuestro proceso pedagógico, de pedagogía crítica; yo empecé a aprender ahí, yo veía sus tableros llenos y yo decía: Ay, Dios mío, qué estoy haciendo aquí sentada. Era como lo que hacíamos, borre, vuelva y haga (Grupo focal ENSQ, comunicación personal, julio de 2018).
\end{abstract}

Habitus que, para el caso de las normales, se evidenció en el trabajo social dado que les dio la posibilidad a los estudiantes, especialmente a aquellos con tendencia a ser maestros, de interactuar y acercarse a diferentes poblaciones, niveles y grados, rurales y urbanos, como una forma de práctica pedagógica (González, Aguirre y Duque, 2005). Habitus, a través de la capacidad adquirida mediante un trabajo pedagógico de inculcación rutinizada, suficiente para producir y reproducir en los destinatarios legítimos, en los estudiantes futuros maestros, una formación en tres grados, con elementos distintivos o de identificación común.

Primero, prácticas pedagógicas homogéneas y duraderas, conforme a los principios de la arbitrariedad inculcada. Segundo, una formación transferible, "capaz de engendrar prácticas conformes a los principios de la arbitrariedad inculcada al mayor número de campos posibles" (Bourdieau y Passeron, 1996, p. 74), como campo educativo, intelectual, artístico, cultural, social, religioso, económico, entre otros. El siguiente testimonio, lo ejemplifica:

Y hacíamos en ese entonces la práctica en la primaria. Yo recuerdo que como a las cinco de la tarde entramos con los profes de primaria, explicábamos con los muchachos cómo se iba a planear, qué iban a hacer y los mismos muchachos al tablero y nosotras ahí para que ellos vinieran a dar la práctica y siempre el acompañamiento en la normal... 
todas trabajamos en la mañana, en la tarde y en la noche (Grupo focal ENSQ, comunicación personal, julio de 2018).

Y tercero, una formación exhaustiva, "que reproduce más completamente en las prácticas, que engendra los principios de la arbitrariedad cultural de un grupo o de una clase" (Bourdieau y Passeron, 1996, p. 75). Interiorización de inculcación rutinizada, desde el trabajo pedagógico del maestro formador del CFC, que fue capaz de perpetuarse una vez finalizaba la arbitrariedad pedagógica y de evidenciarse según "el grado de realización cultural o grado de competencia legítima" (Bourdieau y Passeron, 1996, p. 75) en los sitios de práctica pedagógica, tanto al interior de la normal como en los lugares de convenio, efectivamente guiados por el maestro formador. El siguiente argumento, lo ilustra:

Generalmente uno empieza imitando al maestro, yo empecé a los siete años imitando el mío y naturalmente que tenía otro maestro, imitaba ese otro maestro, uno tiene la tendencia a imitar siempre al maestro que le parece mejor, más alternativo y a no imitar y a evitar rasgos del maestro violento o del maestro autoritario, lo que uno hace toda la vida es reconfigurarse como reubicarse otra vez $(\mathrm{G}$, Arias, comunicación personal, septiembre de 2018).

Por otra parte, el currículo problematizador, propuesto años atrás en la normales, posibilitó, en algunas del eje cafetero, el surgimiento de prácticas pedagógicas distintas a las legitimadas en la Escuela Normal desde la normatividad institucional, pero con algunas características distintivas, propias de la institución. Estas fueron las prácticas pedagógicas investigativas, con carácter transformador de realidades, a través de la solución de problemas educativos desde competencias personales, las cuales ubicaron al maestro como investigador y observador de la realidad educativa; y desde competencias administrativas de gestión y liderazgo con miras a describir, interpretar o transformar la realidad educativa (García, 2006).

Fue desde la práctica pedagógica investigativa desde donde con impulso, se fundamentó la solución de problemas, a partir del trabajo en equipo, de duplas, normalista y maestro en ejercicio, normalista - normalista, maestro formador con maestro formador, en espacios comunes donde se comunicaban experiencias, deseos, se llegó a acuerdos o desacuerdos y se resolvieron conjuntamente problemas (Sáenz, 2010). La práctica pedagógica fue el espacio donde los maestros en formación y los maestros en ejercicio conjuntamente construían, implícita o explícitamente, aprendizajes por medio del trabajo pedagógico centrado en problemas concretos, producto del análisis de situaciones reales.

Las prácticas pedagógicas estaban basadas en el espíritu investigativo, nos iniciábamos en los proyectos de investigación y las prácticas se volvieron prácticas pedagógicas investigativas; no era solo la práctica docente sino que los practicantes, los estudiantes de la Normal desde 
grado octavo se iniciaban en unos reconocimientos en los ambientes educativos porque en grado 11 recibían el título de bachiller con profundización en pedagogía y luego venía el ciclo complementario, primero se le llamó grado 12 y 13 pero luego se volvió ciclo de formación complementaria y las prácticas estaban a partir de proyectos de investigación. Cada Normal hizo lo que se llamó el sistema institucional de investigación que integraba las prácticas y la investigación que hacían los estudiantes para poderse graduar y algunos ejercicios con los cuales se vinculaban los docentes. Entonces las normales dieron un tránsito muy grande hacia problematizar la práctica pedagógica (G. Arias, comunicación personal, septiembre de 2018).

\section{Al respecto, Bourdieu (1993), expresa que:}

Existió una relación indisociable entre el habitus de clase o de grupo y el habitus individual [del maestro]; se podría considerar que el habitus individual refleja o expresa objetivamente el habitus del grupo [de la Escuela Normal] y constituye un sistema de disposición individual, una variante estructural [en los maestros], en el que se expresa la singularidad, tanto de la posición en el interior de la [Normal] como de las trayectorias sociales. Es así como el poder de selección que da la experiencia, puede desviar el estilo propio de una época o una clase y, por ende, dar infinidad de diferencias singulares (Bourdieu, 1993, p.104).

En ese sentido, el trabajo pedagógico de inculcación rutinizada propició en los maestros formadores de maestros del CFC, o bien prácticas pedagógicas distintivas, de grupo, reproductoras, con características propias institucionales o bien prácticas pedagógicas distintas, individuales, al trascurrir en las prácticas pedagógicas investigativas. Prácticas pedagógicas que contaron con esquemas y principios de clasificación, de división, que legitimaron y distinguieron lo objetivamente bueno de lo malo a través de la normatividad institucional legitimada en manuales de práctica pedagógica, en instructivos de práctica, en actas de reuniones. En conclusión, lo que hizo posible la diferenciación de estas, fueron las elecciones que los maestros hicieron, su sentido práctico, puesto que los principios pudieron ser o no diferentes para unos y otros maestros (Bourdieu, 1997). El siguiente testimonio, lo ejemplifica:

Cuando tenía Anexa y todos los maestros de formación hacían su práctica y se determinaba todo lo que era la práctica, todo lo que era el proceso normalista en la Anexa. Nosotros en la Anexa teníamos, pues, toda la autoridad y toda la autonomía para determinar esa práctica, no eran los profesores de allá, no, éramos nosotros los de la Anexa, nosotros explicábamos los fundamentos, ehh, como los procesos de cada una, las materias, las jornadas de planeación, cada uno de nosotros hacíamos el proceso, nosotros ganábamos el 15\% de sobresueldo por hacer ese proceso, si (L. Cortés, comunicación personal, julio de 2018). 
Finalizando la primera década del siglo XXI, se plantearon currículos organizados alrededor de proyectos de investigación como ejes en torno a los cuales se programó la acción pedagógica institucional, a partir de actividades propias de los campos de formación que estructuraron el plan de estudios, mediados por principios metodológicos como la integración teoría- práctica, la flexibilidad curricular, la autodirección en el aprendizaje y la participación activa; proyectos previamente diseñados por colectivos académicos, por grupos de maestros formadores de maestros, para responder a necesidades, demandas y problemas de interés propios de las prácticas pedagógicas, legitimados en la normatividad institucional, en el PEI (Pineda-Rodríguez y Loaiza-Zuluaga, 2018; Ramírez, 2008).

Cabe anotar que el modelo pedagógico, en una escuela normal, fue el encargado de normalizar y reglamentar el proceso educativo que definió qué se debía aprender, desde el plan de estudios, con respecto a la formación de maestros en términos del ser, conocer, hacer y convivir; quiénes debían aprender, dentro de la comunidad educativa; quiénes orientaban el aprendizaje, en el ámbito escolar y no escolar con base en el trabajo pedagógico de inculcación rutinizada a través del habitus; con qué procedimientos, metodologías y didácticas se operacionalizaba el currículo; en qué momentos, tiempos y espacios; con qué reglas de funcionamiento académico y de convivencia; cómo se evaluaba el proceso de enseñanza y de aprendizaje y bajo qué parámetros se regulaba la producción de la cultura escolar (Morales, 2010).

En consecuencia, un modelo pedagógico podría asemejarse a lo que Bourdieu denominó habitus cultivado y que funcionó como un instrumento de traducción, como un efecto automático de la pertenencia al campo, en otros contextos con idénticas condiciones sociales (Bourdieu, 2002).

Modelo pedagógico que, constantemente, estructuró el trabajo pedagógico de inculcación rutinizada o quehacer del maestro formador de maestros en dos ámbitos: el primero, caracterizado por ser poco directivo pero muy afectivo, con actitudes afiliativas, orientador y asesor de estudiantes, mediador analítico- hermeneútico de procesos de enseñanza en contexto, sobre los cuales diseñaba la arquitectura de los conocimientos, con base en los símbolos apropiados para el aprendizaje y un mediador práctico, basado en un quehacer ético y eficiente. Y el segundo maestro, formador de formadores, un agente activo en la búsqueda de información, capaz de trabajar colectivamente en equipo (Morales, 2010). El siguiente testimonio, lo explicita:

En la Normal hubo, para mí, yo creo que de los maestros más expertos en pedagogía que le aprendí mucho, se llama Gustavo Arias, él maneja la parte de la pedagogía. Y tuvimos la oportunidad de traernos un profesor que estuvo explicando todo el modelo de Cuba, entonces frente a eso y digo que fue más la parte de las capacitaciones $(\mathrm{G}$, Mendieta, comunicación personal, septiembre de 2018). 
El habitus, permitió, por tanto, con base en los postulados de Bourdieau (1993): "producir un número infinito de prácticas [pedagógicas], individuales y colectivas, relativamente imprevisibles pero limitadas en su diversidad, capaces de excluir, sin violencia, todas las acciones incompatibles con las condiciones objetivadas institucionalmente" (Bourdieu, 1993, p. 97). Afirmación que se ve reflejada en el siguiente testimonio:

La maestra de investigación tiene una mirada tan holística porque tiene que tener contacto con todas las disciplinas y con todos los problemas de la enseñabilidad de la disciplina. Una evaluación del aprendizaje, una evaluación con los niños, cómo los niños que aprenden a leer y a escribir significativamente, que tiene que ver con el pensamiento numérico, qué tiene que ver con la construcción de ciudadanía, qué tiene que ver la investigación con eso, porque tu no das una investigación formal, teórica, no, es en contexto y los contextos son disciplinares y con sujetos reales en una práctica completa identificando problemas concretos, entonces mira que eso le da una mirada a uno muy preocupante porque cada vez es más fuerte, cómo lo hago, eso cambia el maestro (L, Uribe, comunicación personal, julio de 2018).

\section{Conclusiones}

La presente investigación tuvo como objetivo presentar los resultados de la investigación denominada Prácticas pedagógicas que caracterizaron el quehacer del maestro en el ciclo de formación complementaria de las Escuelas Normales del eje cafetero, 1994-2010.

Se concluye, que las políticas educativas reglamentadas a través de la normatividad, dispuesta por parte de la máxima autoridad pedagógica o Ministerio de Educación Nacional, legitimaron, reestructuraron y acreditaron las escuelas normales y con ellas, las prácticas pedagógicas de los maestros del CFC; proceso viabilizado por las instancias pedagógicas o secretarías de educación, departamentales y municipales.

Cada contexto educativo, si bien estuvo amparado por la legislación nacional, estableció su propio canon de formación legitimado en la normatividad institucional, reflejado en el modelo pedagógico, el currículo, el plan de estudios, dentro del PEI, como la acción pedagógica institucional, a partir de la cual, se configuró el trabajo pedagógico de inculcación rutinizada, colectivo e individual, el cual, desde diferentes tipos de habitus, posibilitó un sistema integrado de quehaceres y discursos frente al saber pedagógico y a las realidades del mismo en los diversos escenarios pedagógicos.

La incorporación de estructuras rutinizadas o habitus generadores de nuevos habitus, posibilitó cultivar o mantener principios comunes de prácticas pedagógicas y asegurar prácticas pedagógicas rutinizadas, con acciones 
comunes, distintivas de cada Normal; o, por el contrario, prácticas pedagógicas con acciones diferenciadas, distintas a las habitualmente constituidas, dados los acercamientos a procesos de investigación pedagógica de los que fueron partícipes.

Finalmente, a manera de colofón, con el presente análisis, se espera contribuir al debate académico sobre la formación de los maestros en la región Eje Cafetero y del país, desde el vínculo Universidad-Escuelas Normales y por ende, a la cualificación de los actuales Programas de Formación Complementaria en los procesos de formación; aportar al conocimiento científico en la historia del maestro colombiano, dado que caracteriza las prácticas pedagógicas en la formación de maestros normalistas con base en las complejas relaciones sociales, políticas y pedagógicas que se han presentado en el sujeto del saber pedagógico; además, aportar al historial de práctica pedagógica, sus principios, precedentes y narraciones, enseñanzas disponibles para maestros activos y nuevos, que podrían constituir redes académicas para la difusión de discusión e innovación sobre las prácticas pedagógicas de los maestros formadores de maestros.

\section{Referencias Bibliográficas}

Ávila, R. (2007). Fundamentos de pedagogía, hacia una comprensión del saber pedagógico. Bogotápá Cooperativa Editorial Magisterio.

Bourdieau, P., \& Passeron, J. C. (1996). La reproducción, elementos para una teoría del sistema de enseñanza. México: Fontamara.

Bourdieu, P. (1993). El sentido práctico. Madrid: Taurus.

Bourdieu, P. (1997). Razones prácticas sobre la teoría de la acción. Bercelona: Anagrama.

Bourdieu, P. (2002). Campo de poder, campo intelectual. Argentina: Monstressor, Jungla simbólica.

Cajamarca, C. E. (1992). Proyecto pedagógico, formación integral con base en procesos y valores. [Informe]. Bogotá: s. ed.

Cardoso, C. F. (2000). Introducción al trabajo de la investigación histórica, conocimiento, método e historia. España: Crítica Barcelona.

Escuela Normal Superior de Manizales. (31 de enero de 1997). Acta No. 02 de reunión de profesores. [Manuscrito no publicado]. Manizales, Caldas, Colombia: Escuela Normal Superior de Manizales.

Escuela Normal Superior de Manizales. (febrero de 2002). Circular para docentes. [Manuscrito no publicado]. Manizales, Caldas, Colombia: Escuela Normal Superior de Manizales. 
Escuela Normal Superior de Manizales. (17 de febrero de 2004). Acta comité de práctica pedagógica investigativa. [Manuscrito no publicado]. Manizales, Caldas, Colombia: Escuela Normal Superior de Manizales.

García, M. E. (mayo de 2006). Informe. Práctica pedagógica investigativa en atención educativa a grupos poblacionales. [Manuscrito no publicado]. Manizales, Caldas, Colombia: Escuela Normal Superior de Manizales.

González, M. E., Aguirre, M. M., y Duque, M. C. (abril de 2005). Informe General de la Unidad de Apoyo Integral (UAI). [Manuscrito no publicado]. Marquetalia, Caldas, Colombia: Escuela Normal Suerior Nuestra Señora de la Candelaria.

Jaramillo, L., Candamil, Á., y Ocampo, G. (1999). Manual de funciones y requisitos. [Manuscrito no publicado]. Manizales, Caldas, Colombia: Escuela Normal Superior de Manizales.

Lloreda, F. J. (julio de 2002). Evaluación externa con fines de acreditación de calidad y desarrollo de las escuelas normales superiores. Documento del grupo de Formación y Evaluación de Educadores de la Dirección de Calidad de la Educación Preescolar, Básica y Media del Ministerio de Educación Nacional. Bogotá, Cundinamarca, Colombia: Ministerio de Educación Nacional.

Loaiza, Y. E., Pineda, Y. L., y Arbeláez, N. (2014). Debate pedagógico: El maestro de las escuelas normales de Manizales en el contexto del movimiento pedagógico colombiano. Manizales: Universidad de Caldas.

López, M. A., Tobos, M. E., y Baldovino, M. M. (13 de noviembre de 2003). Evaluación externa con fines de acreditación de calidad y desarrollo. Escuela Normal Superior del Risaralda. [Manuscrito no publicado]. Pereira, Risaralda, Colombia: Ministerio de Educación Nacional.

Ministerio de Educación Nacional. (julio de 2002). Evaluación externa con fines de acreditación de calidad y desarrollo, escuelas normales superiores. Grupo de Formación y Evaluación de Educadores de la Dirección de Calidad de la Educación Preescolar, Básica y Media. Bogotá, Cundinamarca, Colombia: Ministerio de Educación Nacional.

Morales, J. (enero de 2010). Informe: Pertinencia del programa de formación complementaria. [Manuscrito no publicado]. Manizales, Caldas, Colombia: Escuela Normal Superior de Manizales.

Nieto, L. V. (mayo de 1998). Documento de trabajo del Sistema Nacional de Formación de Educadores: Proceso de acompañamiento para la acreditación de las ENS. Seminario taller El Proyecto Educativo Institucional (PEI). Bogotá, Cundinamarca, Colombia: Ministerio de Educación Nacional.

Ocampo, J. D., y Espitia, M. (2001). I Encuentro Subregional de Escuelas Normales del Sur Occidentes Colombiano. Pereira: Escuela Normal Superior del Risaralda.

Pineda-Rodríguez, Y., y Loaiza-Zuluaga, Y. (2017). Un análisis del trayecto histórico del currículo en Colombia. Segunda mitad del siglo XX. Revista de 
Investigaciones UCM, 17(29), 150-167. http://dx.doi.org/10.22383/ri.v17i29.94

Pineda-Rodríguez, Y., y Loaiza-Zuluaga, Y. (2018). Estado del arte de las prácticas pedagógicas de los maestros de las escuelas normales superiores y las facultades de educación. PRAXIS, 14(2), 265-285. DOI: http://dx.doi.org/10.21676/23897856.2914

Ramírez, M. I. (2008). Informe de procesos investigativos. [Manuscrito no publicado]. Manizales, Caldas, Colombia: Escuela Normal Superior de Manizales.

Sáenz O., C. (2010). Proyecto Educativo Institucional. [Manuscrito no publicado]. Anserma, Caldas, Colombia: Escuela Normal Superior Rebeca Sierra.

Valencia, C. H. (2006). Las escuelas normales y la formación del magisterio, primera mitad del siglo XX. Manizales: Universidad de Caldas. 